\title{
POSITIVE SOLUTIONS OF QUASILINEAR ELLIPTIC EQUATIONS WITH EXPONENTIAL NONLINEARITY COMBINED WITH CONVECTION TERM
}

\author{
ANDERSON L.A. DE ARAUJO AND LUIZ F.O FARIA
}

\begin{abstract}
We establish the existence of positive solutions for a nonlinear elliptic Dirichlet problem in dimension $N$ involving the $N$-Laplacian. The nonlinearity considered depends on the gradient of the unknown function and an exponential term. In such case, variational methods cannot be applied. Our approach is based on approximation scheme, where we consider a new class of normed spaces of finite dimension. As a particular case, we extended the result achieved by De Araujo and Montenegro [2016] for any $N>2$.
\end{abstract}

\section{INTRODUCTION}

Let $\Omega \subset \mathbb{R}^{N}$ be a smooth bounded domain and $p>1$. Consider the following problem

$$
\begin{cases}-\Delta_{p} u=g(x, u, \nabla u) & \text { in } \quad \Omega \\ u=0 & \text { on } \partial \Omega .\end{cases}
$$

Here, the operator $-\Delta_{p}: W_{0}^{1, p}(\Omega) \rightarrow W^{-1, p^{\prime}}(\Omega)$, where $\frac{1}{p}+\frac{1}{p^{\prime}}=1$, is defined by

$$
\left\langle-\Delta_{p} u, v\right\rangle=\int_{\Omega}|\nabla u|^{p-2} \nabla u \nabla v d x \text { for all } u, v \in W_{0}^{1, p}(\Omega),
$$

and the forcing term $g$ has the form of a convection term, that is, it depends also on the gradient of the unknown function. Due to the presence of the gradient $\nabla u$ in the term $g(x, u, \nabla u)$, problem (1.1) does not have, in general, variational structure. This kind of problems are usually studied by means of topological degree, the method of sub-supersolutions, fixed point theory, approximation techniques and iterative scheme. For instance, we would like to cite $[2,5,8,12-14,25]$. In particular, in [13], via an approximation on finite dimensional subspaces, the authors proved the existence of a positive solution for the following problem

$$
\begin{cases}-\Delta_{p} u-\mu \Delta_{q} u=g(x, u, \nabla u) & \text { in } \Omega, \\ u>0 & \text { in } \Omega, \\ u=0 & \text { on } \partial \Omega\end{cases}
$$

where $\mu \geq 0, g: \Omega \times \mathbb{R} \times \mathbb{R}^{N} \rightarrow \mathbb{R}$ is a continuous function satisfying the growth condition:

(G) $b_{0}|t|^{r_{0}} \leq f(x, t, \xi) \leq b_{1}\left(1+|t|^{r_{1}}+|\xi|^{r_{2}}\right)$ for all $(x, t, \xi) \in \Omega \times \mathbb{R} \times \mathbb{R}^{N}$, with constants $b_{0}, b_{1}>0, r_{1}, r_{2} \in[0, p-1), r_{0} \in[0, p-1)$ if $\mu=0$, and $r_{0} \in[0, q-1]$ if $\mu>0$.

2010 Mathematics Subject Classification. 35B33; 35J62; 35J66; 35J92; 37L65.

Key words and phrases. Dirichlet problem for the $N$-Laplacian; Galerkin approximation; Trudinger-Moser inequality; exponential growth; convection term.

Luiz F.O Faria was partially supported by FAPEMIG CEX APQ 02374/17. 
On the other hand, elliptic problems of the type

$$
\begin{cases}-\Delta_{N} v=g(x, v) & \text { in } \quad \Omega \\ v=0 & \text { on } \partial \Omega\end{cases}
$$

where $\Omega \subset \mathbb{R}^{N}$ and $g(x, v)$ is continuous and behaves like $\exp \left(\alpha|v|^{N /(N-1)}\right)$ as $|v| \rightarrow+\infty$ have been studied by many authors, we would like to cite $[3,6,9-11,19,26]$. One of the main ingredients is the Trudinger-Moser inequality introduced in [22,28]. Namely, given $u \in W_{0}^{1, N}(\Omega)$, then

$$
e^{\sigma|u|^{\frac{N}{N-1}}} \in L^{1}(\Omega) \text { for every } \sigma>0,
$$

and there exists a positive constant $L(N)$ which depends on $N$ only, such that

$$
\sup _{\|u\|_{W_{0}^{1, N}(\Omega)} \leq 1} \int_{\Omega} e^{\sigma|u|^{\frac{N}{N-1}}} d x \leq L(N)|\Omega| \text { for every } \sigma \leq \alpha_{N},
$$

where $|\Omega|=\int_{\Omega} d x, \alpha_{N}=N w_{N-1}^{\frac{1}{N-1}}$ and $w_{N-1}$ is the $(N-1)$-dimensional measure of the $(N-1)$-sphere.

In particular, in [6] the authors proved existence of solutions for the following problem

$$
\begin{cases}-\Delta u=\lambda u^{q}+f(u) & \text { in } \Omega, \\ u>0 & \text { in } \Omega, \\ u=0 & \text { on } \partial \Omega,\end{cases}
$$

where $\Omega \subset \mathbb{R}^{2}, \lambda>0$ is a small enough parameter, $0<q<1$ and $f:[0, \infty) \rightarrow \mathbb{R}$ is a continuous function satisfying the growth condition:

$(H) 0 \leq t f(t) \leq C|t|^{r} \exp \left(\alpha t^{2}\right)$ where $\alpha>0$ and $r>2$.

In [7], still considering $N=2$, the authors proved existence of solutions for an elliptic system with arguments based in [6] and with nonlinearities satisfying the growth condition $(H)$.

In this work we are concerned with the existence of positive solutions for the problem:

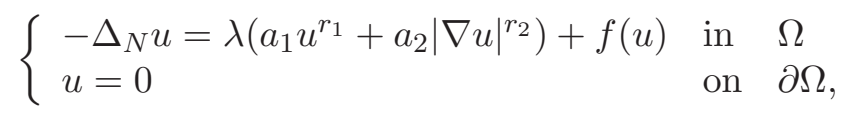

where $\Omega$ in $\mathbb{R}^{N}$ is a bounded domain with a $C^{1, \alpha}$-boundary $\partial \Omega$, for some $0<\alpha \leq 1, \lambda>0$ is a parameter, $0<r_{i}<N-1$, for $i=1,2, a_{1}>0, a_{2} \geq 0$, and $f:[0, \infty) \rightarrow \mathbb{R}$ is a nonegative continuous function. The main assumption on the function $f$ is the following, which will be referred throughout the paper as $(F)$ :

$(F) 0 \leq t f(t) \leq a_{3} t^{r_{3}+1} \exp \left(\alpha t^{\frac{N}{N-1}}\right)$ where $a_{3}, \alpha>0$, and $r_{3}>N-1$.

Most of the papers, to prove existence results for the problem, assume AmbrosettiRabinowitz conditions (or some additional conditions) to obtain Palais-Smale or Cerami compactness condition. Notice that in this paper we don't need to impose such extra hypotheses.

An interesting problem related to $(\mathrm{P})$, by considering a more general operator, was treated by [29]. The authors studied a $(N, q)$-Laplacian problem with a critical Trundinger-Moser 
nonlinearity as following

$$
\begin{cases}-\Delta_{N} u-\Delta_{q} u=\mu|u|^{q-2} u+\lambda|u|^{N-2} u e^{|u|^{N /(N-1)}} & \text { in } \quad \Omega \\ u=0 & \text { on } \partial \Omega\end{cases}
$$

where $N>q>1, \mu \in \mathbb{R}$ and $\lambda>0$. By using a critical point theorem, based on a cohomological index, they proved the existence of solution for $\mu$ interacting with the first eigenvalue of the $\left(-\Delta_{q} u, W_{0}^{1, q}(\Omega)\right)$ operator and for $\lambda$ sufficiently large.

Here we extend the results of [6] for the general dimension case $N>1\left(a_{2}=0\right)$. In order to prove the existence of positive solutions for $(P)$, we borrow some ideas from [6] and [13]. Due to the presence of the supercritical term $\exp \left(\alpha|v|^{N /(N-1)}\right)$, along with the convection term, we had to overcome some problems. For example, in $W_{0}^{1, N}(\Omega)$ we need to assume a Schauder basis instead of the Hilbert basis (like in [6]), which becomes some additional difficulty. By comparing with [13], due to the presence of the term $\exp \left(\alpha|v|^{N /(N-1)}\right)$, a suitable modification on the approximating approach had to be done. Although in [13] the authors used the Schauder basis, we could not obtain the necessary estimates for this approach by considering the approximate spaces used there. To do this, we consider a new class of normed spaces of finite dimension.

Our main result reads as follows:

Theorem 1.1. Suppose that $f:[0, \infty) \rightarrow \mathbb{R}$ is a continuous function satisfying the assumption $(F)$. Then there exists $\lambda^{*}>0$ such that for every $\lambda \in\left(0, \lambda^{*}\right)$ problem $(P)$ admits a (positive) weak solution $u \in W_{0}^{1, N}(\Omega)$.

\section{Preliminary Results}

The Sobolev space $W_{0}^{1, N}(\Omega)$ is endowed with the norm

$$
\|u\|_{W_{0}^{1, N}(\Omega)}=\left(\int_{\Omega}|\nabla u|^{N} d x\right)^{1 / N} .
$$

To prove Theorem 1.1 we approximate $f$ by Lipschitz functions $f_{k}: \mathbb{R} \rightarrow \mathbb{R}$ defined by

$$
f_{k}(s)=\left\{\begin{array}{llc}
-k\left[G\left(-k-\frac{1}{k}\right)-G(-k)\right], & \text { if } & s \leq-k, \\
-k\left[G\left(s-\frac{1}{k}\right)-G(s)\right], & \text { if } \quad-k \leq s \leq-\frac{1}{k}, \\
k^{2} s\left[G\left(-\frac{2}{k}\right)-G\left(-\frac{1}{k}\right)\right], & \text { if } \quad-\frac{1}{k} \leq s \leq 0, \\
k^{2} s\left[G\left(\frac{2}{k}\right)-G\left(\frac{1}{k}\right)\right], & \text { if } \quad 0 \leq s \leq \frac{1}{k}, \\
k\left[G\left(s+\frac{1}{k}\right)-G(s)\right], & \text { if } \quad \frac{1}{k} \leq s \leq k, \\
k\left[G\left(k+\frac{1}{k}\right)-G(k)\right], & \text { if } \quad s \geq k,
\end{array}\right.
$$

where $G(s)=\int_{0}^{s} f(\xi) d \xi$.

The following (approximation) result was proved in [27] and uses the explicit expression of the sequence (2.1).

Lemma 2.1. Let $f: \mathbb{R} \rightarrow \mathbb{R}$ be a continuous function such that $s f(s) \geq 0$ for every $s \in \mathbb{R}$. Then there exists a sequence $f_{k}: \mathbb{R} \rightarrow \mathbb{R}$ of continuous functions satisfying

(i) $s f_{k}(s) \geq 0$ for every $s \in \mathbb{R}$;

(ii) $\forall k \in \mathbb{N} \exists c_{k}>0$ such that $\left|f_{k}(\xi)-f_{k}(\eta)\right| \leq c_{k}|\xi-\eta|$ for every $\xi, \eta \in \mathbb{R}$;

(iii) $f_{k}$ converges uniformly to $f$ in bounded subsets of $\mathbb{R}$.

The sequence $f_{k}$ of the previous lemma has some additional properties. 
Lemma 2.2. Let $f: \mathbb{R} \rightarrow \mathbb{R}$ be a continuous function satisfying $(F)$ for every $s \in \mathbb{R}$. Then the sequence $f_{k}$ of Lemma 2.1 satisfies

(i) $\forall k \in \mathbb{N}, 0 \leq s f_{k}(s) \leq C_{1}|s|^{r_{3}} \exp \left(2^{\frac{N}{N-1}} \alpha|s|^{\frac{N}{N-1}}\right)$ for every $|s| \geq \frac{1}{k}$;

(ii) $\forall k \in \mathbb{N}, 0 \leq s f_{k}(s) \leq C_{2}|s|^{2} \exp \left(2^{\frac{N}{N-1}} \alpha|s|^{\frac{N}{N-1}}\right)$ for every $|s| \leq \frac{1}{k}$,

where $C_{1}$ and $C_{2}$ are positive constants independent of $k$.

Proof. Everywhere in this proof the constant $a_{3}$ is the one of (2.1).

First step. Suppose that $-k \leq s \leq-\frac{1}{k}$.

By the mean value theorem, there exists $\eta \in\left(s-\frac{1}{k}, s\right)$ such that

$$
f_{k}(s)=-k\left[G\left(s-\frac{1}{k}\right)-G(s)\right]=-k G^{\prime}(\eta)\left(s-\frac{1}{k}-s\right)=f(\eta)
$$

and

$$
s f_{k}(s)=s f(\eta) .
$$

Since $s-\frac{1}{k}<\eta<s<0$ and $f(\eta)<0$, we have $s f(\eta) \leq \eta f(\eta)$. Therefore,

$$
\begin{aligned}
s f_{k}(s) \leq \eta f(\eta) & \leq a_{3}|\eta|^{r_{3}} \exp \left(\alpha|\eta|^{\frac{N}{N-1}}\right) \\
& \leq a_{3}\left|s-\frac{1}{k}\right|^{r_{3}} \exp \left(\alpha\left|s-\frac{1}{k}\right|^{\frac{N}{N-1}}\right) \\
& \leq a_{3}\left(|s|+\frac{1}{k}\right)^{r_{3}} \exp \left(\alpha\left(|s|+\frac{1}{k}\right)^{\frac{N}{N-1}}\right) \\
& \leq a_{3}(2|s|)^{r_{3}} \exp \left(\alpha(2|s|)^{\frac{N}{N-1}}\right) \\
& =a_{3} 2^{r_{3}}|s|^{r_{3}} \exp \left(2^{\frac{N}{N-1}} \alpha|s|^{\frac{N}{N-1}}\right) .
\end{aligned}
$$

Second step. Assume $\frac{1}{k} \leq s \leq k$.

By the mean value theorem, there exists $\eta \in\left(s, s+\frac{1}{k}\right)$ such that

$$
f_{k}(s)=k\left[G\left(s+\frac{1}{k}\right)-G(s)\right]=k G^{\prime}(\eta)\left(s+\frac{1}{k}-s\right)=f(\eta)
$$

and

$$
s f_{k}(s)=s f(\eta) .
$$

Since $0<s<\eta<s+\frac{1}{k}$ and $f(\eta)>0$, we have $s f(\eta) \leq \eta f(\eta)$. Therefore,

$$
\begin{aligned}
s f_{k}(s) \leq \eta f(\eta) & \leq a_{3}|\eta|^{r_{3}} \exp \left(\alpha|\eta|^{\frac{N}{N-1}}\right) \\
& \leq a_{3}\left|s+\frac{1}{k}\right|^{r_{3}} \exp \left(\alpha \mid s+\frac{1}{k^{\frac{N}{N-1}}}\right) \\
& \leq a_{3}(2|s|)^{r_{3}} \exp \left(\alpha(2|s|)^{\frac{N}{N-1}}\right) \\
& =a_{3} 2^{r_{3}}|s|^{r_{3}} \exp \left(2^{\frac{N}{N-1}} \alpha|s|^{\frac{N}{N-1}}\right) .
\end{aligned}
$$

Third step. Suppose that $|s| \geq k$, then

$$
f_{k}(s)= \begin{cases}-k\left[G\left(-k-\frac{1}{k}\right)-G(-k)\right], & \text { if } \quad s \leq-k \\ k\left[G\left(k+\frac{1}{k}\right)-G(k)\right], & \text { if } \quad s \geq k .\end{cases}
$$

If $s \leq-k$, by the mean value theorem, there exists $\eta \in\left(-k-\frac{1}{k},-k\right)$ such that

$$
f_{k}(s)=k\left[G\left(-k-\frac{1}{k}\right)-G(-k)\right]=-k G^{\prime}(\eta)\left(-k-\frac{1}{k}-(-k)\right)=f(\eta)
$$

and

$$
s f_{k}(s)=s f(\eta) .
$$


Since $-k-\frac{1}{k}<\eta<-k<0$ and $k<|\eta|<k+\frac{1}{k}$, we conclude that

$$
\begin{aligned}
s f_{k}(s)=\frac{s}{\eta} \eta f(\eta) & \leq \frac{|s|}{|\eta|} a_{3}|\eta|^{r_{3}} \exp \left(\alpha|\eta|^{\frac{N}{N-1}}\right)=a_{3}|s||\eta|^{r_{3}-1} \exp \left(\alpha|\eta|^{\frac{N}{N-1}}\right) \\
& \leq a_{3}|s|\left(k+\frac{1}{k}\right)^{r_{3}-1} \exp \left(\alpha\left(k+\frac{1}{k}\right)^{\frac{N}{N-1}}\right) \\
& \leq a_{3}|s|\left(|s|+\frac{1}{k}\right)^{r_{3}-1} \exp \left(\alpha\left(|s|+\frac{1}{k}\right)^{\frac{N}{N-1}}\right) \\
& \leq a_{3}|s|(2|s|)^{r_{3}-1} \exp \left(\alpha(2|s|)^{\frac{N}{N-1}}\right) \\
& \leq a_{3} 2^{r_{3}-1}|s|^{r_{3}} \exp \left(2^{\frac{N}{N-1}} \alpha|s|^{\frac{N}{N-1}}\right) .
\end{aligned}
$$

If $s \geq k$, by the mean value theorem, there exists $\eta \in\left(k, k+\frac{1}{k}\right)$ such that

$$
f_{k}(s)=k\left[G\left(k+\frac{1}{k}\right)-G(k)\right]=k G^{\prime}(\eta)\left(k+\frac{1}{k}-k\right)=f(\eta) .
$$

By computations similar to conclude (2.3) one has

$$
s f_{k}(s)=s f(\eta)=\frac{s}{\eta} \eta f(\eta) \leq \frac{|s|}{|\eta|} a_{3}|\eta|^{r_{3}} \exp \left(\alpha|\eta|^{\frac{N}{N-1}}\right) \leq a_{3} 2^{r_{3}-1}|s|^{r_{3}} \exp \left(2^{\frac{N}{N-1}} \alpha|s|^{\frac{N}{N-1}}\right) .
$$

Fourth step. Assume $-\frac{1}{k} \leq s \leq \frac{1}{k}$, then

$$
f_{k}(s)= \begin{cases}k^{2} s\left[G\left(-\frac{2}{k}\right)-G\left(-\frac{1}{k}\right)\right], & \text { if } \quad-\frac{1}{k} \leq s \leq 0 \\ k^{2} s\left[G\left(\frac{2}{k}\right)-G\left(\frac{1}{k}\right)\right], & \text { if } \quad 0 \leq s \geq \frac{1}{k} .\end{cases}
$$

If $-\frac{1}{k} \leq s \leq 0$, by the mean value theorem, there exists $\eta \in\left(-\frac{2}{k},-\frac{1}{k}\right)$ such that

$$
f_{k}(s)=k^{2} s\left[G\left(-\frac{2}{k}\right)-G\left(-\frac{1}{k}\right)\right]=k^{2} s G^{\prime}(\eta)\left(-\frac{2}{k}-\left(-\frac{1}{k}\right)\right)=-k s f(\eta) .
$$

Therefore

$$
\begin{gathered}
s f_{k}(s)=-k s^{2} f(\eta)=-k \frac{s^{2}}{\eta} \eta f(\eta) \leq k \frac{s^{2}}{|\eta|} \eta f(\eta) \leq \\
\leq a_{3} k|s|^{2}|\eta|^{r_{3}-1} \exp \left(\alpha|\eta|^{\frac{N}{N-1}}\right) \leq a_{3} k|s|^{2}\left(\frac{2}{k}\right)^{r_{3}-1} \exp \left(\alpha|\eta|^{\frac{N}{N-1}}\right) \leq a_{3} 2^{r_{3}-1}|s|^{2} \exp \left(\alpha\left(\frac{2}{k}\right)^{\frac{N}{N-1}}\right) \\
(2.5) \quad \leq a_{3} 2^{r_{3}-1}|s|^{2} \exp \left(2^{\frac{N}{N-1}} \alpha\right) \leq a_{3} 2^{r_{3}-1} \exp \left(2^{\frac{N}{N-1}} \alpha\right)|s|^{2} \exp \left(2^{\frac{N}{N-1}} \alpha|s|^{\frac{N}{N-1}}\right) .
\end{gathered}
$$

If $0 \leq s \leq \frac{1}{k}$, by the mean value theorem, there exists $\eta \in\left(\frac{1}{k}, \frac{2}{k}\right)$ such that

$$
f_{k}(s)=k^{2} s\left[G\left(\frac{2}{k}\right)-G\left(\frac{1}{k}\right)\right]=k^{2} s G^{\prime}(\eta)\left(\frac{2}{k}-\frac{1}{k}\right)=k s f(\eta) .
$$

By similar computations to conclude (2.5) one obtains

$$
s f_{k}(s)=k s^{2} f(\eta)=k \frac{s^{2}}{|\eta|} \eta f(\eta) \leq a_{3} 2^{r_{3}-1} \exp \left(2^{\frac{N}{N-1}} \alpha\right)|s|^{2} \exp \left(2^{\frac{N}{N-1}} \alpha|s|^{\frac{N}{N-1}}\right) .
$$

The proof of the lemma follows by taking $C_{1}=a_{3} 2^{r_{3}}$ ad $C_{2}=a_{3} 2^{r_{3}-1} C 2^{r_{3}-1} \exp \left(2^{\frac{N}{N-1}} \alpha\right)$ where $a_{3}$ is given in $(F)$.

Before concluding this section, we will enunciate a comparison principle due to Faria, Miyagaki and Motreanu [13, Theorem 2.2].

Consider the Dirichlet problem

$$
\begin{cases}-\Delta_{p} u-\mu \Delta_{q} u=g(u) & \text { in } \quad \Omega \\ u=0 & \text { on } \quad \partial \Omega\end{cases}
$$


where $1<q \leq p<+\infty, \mu \geq 0$ and $g: \mathbb{R} \rightarrow \mathbb{R}$ is a continuous function.

We recall that $u_{1} \in W^{1, p}(\Omega)$ is a subsolution of problem (2.6) if $u_{1} \geq 0$ a.e. on $\partial \Omega$ and

$$
\int_{\Omega}\left(\left|\nabla u_{1}\right|^{p-2} \nabla u_{1} \nabla \varphi+\mu\left|\nabla u_{1}\right|^{q-2} \nabla u_{1} \nabla \varphi\right) d x \leq \int_{\Omega} g\left(u_{1}\right) \varphi d x
$$

for all $\varphi \in W_{0}^{1, p}(\Omega)$ with $\varphi \geq 0$ a.e. in $\Omega$, while $u_{2} \in W^{1, p}(\Omega)$ is a supersolution of (2.6) if the reversed inequalities are satisfied with $u_{2}$ in place of $u_{1}$ for all $\varphi \in W_{0}^{1, p}(\Omega)$ with $\varphi \geq 0$ a.e. in $\Omega$.

Theorem 2.3. Let $g: \mathbb{R} \rightarrow \mathbb{R}$ be a continuous function such that $t^{1-q} g(t)$ is nonincreasing for $t>0$ if $\mu>0$, and $t^{1-p} g(t)$ is nonincreasing for $t>0$ if $\mu=0$. Assume that $u_{1} \in$ $W_{0}^{1, p}(\Omega)$ and $u_{2} \in W_{0}^{1, p}(\Omega)$ are a positive subsolution and a positive supersolution of problem (2.6), respectively. If $u_{i} \in L^{\infty}(\Omega) \cap C^{1, \alpha}(\Omega), \Delta_{p} u_{i} \in L^{\infty}(\Omega), u_{i} / u_{j} \in L^{\infty}(\Omega)$ for $i, j=1,2$, then $u_{2} \geq u_{1}$ in $\Omega$.

\section{Approximation problem}

For each $n \in \mathbb{N}$, we define the auxiliary problem $\left(P_{n}\right)$ by

$$
\begin{cases}-\Delta_{N} u=\lambda\left(a_{1}\left(u_{+}\right)^{r_{1}}+a_{2}|\nabla u|^{r_{2}}\right)+f_{n}(u)+\frac{1}{n} & \text { in } \Omega \\ u>0 & \text { in } \Omega \\ u=0 & \text { on } \partial \Omega,\end{cases}
$$

where $f_{n}$ are given by Lemma 2.1 and Lemma 2.2 , and $u_{+}=\max \{u, 0\}$.

To prove Theorem 1.1 we first show the existence of a solution of problem $\left(P_{n}\right)$ by using the Galerkin method. We would like to cite [1] as the seminal paper in this type of approach.

3.1. Finite-Dimensional Spaces. Let $\mathcal{B}=\left\{w_{1}, w_{2}, \ldots, w_{n}, \ldots\right\}$ be a Schauder basis of $W_{0}^{1, N}(\Omega)($ see $[4,15])$. For each positive integer $m$, let

$$
W_{m}=\left[w_{1}, w_{2}, \ldots, w_{m}\right]
$$

be the $m$-dimensional subspace of $W_{0}^{1, N}(\Omega)$ (generated by $\left\{w_{1}, w_{2}, \ldots, w_{m}\right\}$ ) with norm induced from $W_{0}^{1, N}(\Omega)$. Let $\xi=\left(\xi_{1}, \ldots, \xi_{m}\right) \in \mathbb{R}^{m}$, notice that

$$
|\xi|_{m}=\left\|\sum_{j=1}^{m} \xi_{j} w_{j}\right\|_{W_{0}^{1, N}(\Omega)}
$$

defines a norm in $\mathbb{R}^{m}$. In fact, let $\xi^{i}=\left(\xi_{1}^{i}, \ldots, \xi_{m}^{i}\right) \in \mathbb{R}^{m}, i=1,2$, and let $\lambda \in \mathbb{R}$.

(i) $\left|\xi^{1}+\xi^{2}\right|_{m} \leq\left|\xi^{1}\right|_{m}+\left|\xi^{2}\right|_{m}:$

$$
\begin{aligned}
\left|\xi^{1}+\xi^{2}\right|_{m} & =\left\|\sum_{j=1}^{m} \xi_{j}^{1} w_{j}+\sum_{j=1}^{m} \xi_{j}^{2} w_{j}\right\|_{W_{0}^{1, N}(\Omega)} \\
& \leq\left\|\sum_{j=1}^{m} \xi_{j}^{1} w_{j}\right\|_{W_{0}^{1, N}(\Omega)}+\left\|\sum_{j=1}^{m} \xi_{j}^{2} w_{j}\right\|_{W_{0}^{1, N}(\Omega)} \\
& =\left|\xi^{1}\right|_{m}+\left|\xi^{2}\right|_{m} .
\end{aligned}
$$


(ii) $\left|\lambda \xi^{1}\right|_{m}=|\lambda|\left|\xi^{1}\right|_{m}:$

$$
\left|\lambda \xi^{1}\right|_{m}=\left\|\lambda \sum_{j=1}^{m} \xi_{j}^{1} w_{j}\right\|_{W_{0}^{1, N}(\Omega)}=|\lambda|\left\|\sum_{j=1}^{m} \xi_{j}^{1} w_{j}\right\|_{W_{0}^{1, N}(\Omega)}=|\lambda|\left|\xi^{1}\right|_{m} .
$$

(iii) $\left|\xi^{1}\right|_{m}=0 \Leftrightarrow \xi^{1}=0$ :

$(\Rightarrow) 0=\left|\xi^{1}\right|_{m}=\left\|\sum_{j=1}^{m} \xi_{j}^{1} w_{j}\right\|_{W_{0}^{1, N}(\Omega)}$ implies $\sum_{j=1}^{m} \xi_{j}^{1} w_{j}=0$. By uniqueness of the representation (using a Schauder basis) of the null vector, we conclude that $\xi^{1}=0$.

$(\Leftarrow)$ It is trivial.

By using the above notation, we can identify the normed spaces $\left(W_{m},\|\cdot\|_{W_{0}^{1, N}(\Omega)}\right)$ and $\left(\mathbb{R}^{m},|\cdot|_{m}\right)$ by the isometric linear transformation

$$
v=\sum_{j=1}^{m} \xi_{j} w_{j} \in V_{m} \mapsto \xi=\left(\xi_{1}, \ldots, \xi_{m}\right) \in \mathbb{R}^{m} .
$$

The lemma below is a consequence of Brouwers Fixed Point Theorem and its proof can be found in Kesavan [17].

Lemma 3.1. Let $F: \mathbb{R}^{d} \rightarrow \mathbb{R}^{d}$ be a continuous function such that $\langle F(\xi), \xi\rangle \geq 0$ for every $\xi \in \mathbb{R}^{d}$ with $|\xi|=r$ for some $r>0$. Then, there exists $z_{0}$ in the closed ball $\bar{B}_{r}(0)$ such that $F\left(z_{0}\right)=0$.

3.2. Existence. The following result is concerning the existence result for the auxiliary problem $\left(P_{n}\right)$.

Lemma 3.2. There exists $\lambda^{*}>0$ and $n^{*} \in \mathbb{N}$ such that $\left(P_{n}\right)$ admits a (positive) weak solution $v \in W_{0}^{1, N}(\Omega) \cap C^{1, \alpha}(\bar{\Omega})$, for some $0<\alpha<1$, for every $\lambda \in\left(0, \lambda^{*}\right)$ and $n \geq n^{*}$.

Proof. Let $\mathcal{B}=\left\{w_{1}, w_{2}, \ldots, w_{n}, \ldots\right\}$ be a Schauder basis of $W_{0}^{1, N}(\Omega)$. For each positive integer $m$, let $W_{m}=\left[w_{1}, w_{2}, \ldots, w_{m}\right]$. By using the isometric linear transformation (3.1), define the function $F: \mathbb{R}^{m} \rightarrow \mathbb{R}^{m}$ such that $F(\xi)=\left(F_{1}(\xi), F_{2}(\xi), \ldots, F_{m}(\xi)\right)$, where

$$
\begin{aligned}
F_{j}(\xi)= & \int_{\Omega}|\nabla u|^{N-2} \nabla u \nabla w_{j} d x-\lambda\left(a_{1} \int_{\Omega}\left(u_{+}\right)^{r_{1}} w_{j} d x+a_{2} \int_{\Omega}|\nabla u|^{r_{2}} w_{j}\right) d x \\
& -\int_{\Omega} f_{n}\left(u_{+}\right) w_{j}-\frac{1}{n} \int_{\Omega} w_{j} d x, \quad j=1, \ldots, m .
\end{aligned}
$$

Therefore,

$$
\langle F(\xi), \xi\rangle=\int_{\Omega}|\nabla u|^{N} d x-\lambda\left(a_{1} \int_{\Omega}\left(u_{+}\right)^{r_{1}} u d x+a_{2} \int_{\Omega}|\nabla u|^{r_{2}} u d x\right)-\int_{\Omega} f_{n}\left(u_{+}\right) u d x-\frac{1}{n} \int_{\Omega} u d x .
$$

Given $u \in W_{m}$, we define

and

$$
\Omega_{n}^{+}=\left\{x \in \Omega:|u(x)| \geq \frac{1}{n}\right\}
$$

$$
\Omega_{n}^{-}=\left\{x \in \Omega:|u(x)|<\frac{1}{n}\right\}
$$


Thus, we rewrite (3.2) as

$$
\langle F(\xi), \xi\rangle=\langle F(\xi), \xi\rangle_{P}+\langle F(\xi), \xi\rangle_{N}
$$

where

$$
\langle F(\xi), \xi\rangle_{P}=\int_{\Omega_{n}^{+}}|\nabla u|^{N} d x-\lambda\left(a_{1} \int_{\Omega_{n}^{+}}\left(u_{+}\right)^{r_{1}} u d x+a_{2} \int_{\Omega_{n}^{+}}|\nabla u|^{r_{2}} u d x\right)-\int_{\Omega_{n}^{+}} f_{n}\left(u_{+}\right) u_{+} d x-\frac{1}{n} \int_{\Omega_{n}^{+}} u d x
$$

and

$$
\langle F(\xi), \xi\rangle_{N}=\int_{\Omega_{n}^{-}}|\nabla u|^{N} d x-\lambda\left(a_{1} \int_{\Omega_{n}^{-}}\left(u_{+}\right)^{r_{1}} u d x+a_{2} \int_{\Omega_{n}^{-}}|\nabla u|^{r_{2}} u d x\right)-\int_{\Omega_{n}^{-}} f_{n}\left(u_{+}\right) u_{+} d x-\frac{1}{n} \int_{\Omega_{n}^{-}} u d x .
$$

Step 1. Since $0<r_{i}<N-1$, for $i=1,2$, then

$$
\int_{\Omega_{n}^{+}}\left(u_{+}\right)^{r_{1}+1} d x \leq \int_{\Omega}\left(u_{+}\right)^{r_{1}+1} d x \leq \int_{\Omega}|u|^{r_{1}+1} d x=\|u\|_{L^{r_{1}+1}(\Omega)}^{r_{1}+1} \leq C_{1}\|u\|_{W_{0}^{1, N}(\Omega)}^{r_{1}+1} .
$$

By virtue of Lemma 2.2 (i) we get

$$
\begin{aligned}
\int_{\Omega_{n}^{+}} f_{n}\left(u_{+}\right) u_{+} d x & \leq C_{1} \int_{\Omega_{n}^{+}}|u|^{r_{3}+1} \exp \left(2^{\frac{N}{N-1}} \alpha|u|^{\frac{N}{N-1}}\right) d x \\
& \leq a_{3}\left(\int_{\Omega}|u|^{N^{\prime}\left(r_{3}+1\right)} d x\right)^{\frac{1}{N^{\prime}}}\left(\int_{\Omega} \exp \left(N 2^{\frac{N}{N-1}} \alpha|u|^{\frac{N}{N-1}}\right) d x\right)^{\frac{1}{N}} \\
& =a_{3}\|u\|_{L^{N^{\prime}\left(r_{3}+1\right)}(\Omega)}^{r_{3}+1}\left(\int_{\Omega} \exp \left(N 2^{\frac{N}{N-1}} \alpha|u|^{\frac{N}{N-1}}\right) d x\right)^{\frac{1}{N}}
\end{aligned}
$$

where $\frac{1}{N}+\frac{1}{N^{\prime}}=1$.

It follows from (3.3) and (3.4) that

$$
\begin{aligned}
\langle F(\xi), \xi\rangle_{P} \geq & \int_{\Omega_{n}^{+}}|\nabla u|^{N} d x-\lambda\left(a_{1} C_{1}\|u\|_{W_{0}^{1, N}(\Omega)}^{r_{1}+1}+a_{2} \int_{\Omega_{n}^{+}}|\nabla u|^{r_{2}} u d x\right) \\
& -C_{3}\|u\|_{W_{0}^{1, N}(\Omega)}^{r_{3}+1}\left(\int_{\Omega} \exp \left(N 2^{\frac{N}{N-1}} \alpha|u|^{\frac{N}{N-1}}\right) d x\right)^{\frac{1}{N}}-\frac{C_{4}}{n}\|u\|_{W_{0}^{1, N}(\Omega)},
\end{aligned}
$$

where $C_{0}, C_{1}$ and $C_{3}$ are constants not depending $n$ and $m$.

Step 2. Since $0<r_{i}<N-1$, for $i=1,2$, then

$$
\int_{\Omega_{n}^{-}}\left(u_{+}\right)^{r_{1}+1} \leq \int_{\Omega_{n}^{-}}|u|^{r_{1}+1} \leq|\Omega| \frac{1}{n^{r_{1}+1}} .
$$

By virtue of Lemma 2.2 (ii) we get

$$
\int_{\Omega_{n}^{-}} f_{n}\left(u_{+}\right) u_{+} \leq C_{2} \int_{\Omega_{n}^{-}}|u|^{2} \exp \left(2^{\frac{N}{N-1}} \alpha|u|^{\frac{N}{N-1}}\right) d x \leq C_{2} \exp \left(2^{\frac{N}{N-1}} \alpha\right)|\Omega| \frac{1}{n^{2}} .
$$

It follows from (3.6) and (3.7) that

$$
\langle F(\xi), \xi\rangle_{N} \geq \int_{\Omega_{n}^{-}}|\nabla u|^{N}-\lambda\left(a_{1}|\Omega| \frac{1}{n^{r_{1}+1}}+a_{2} \int_{\Omega_{n}^{-}}|\nabla u|^{r_{2}} u d x\right)-C_{3} \exp \left(2^{\frac{N}{N-1}} \alpha\right)|\Omega| \frac{1}{n^{2}}-|\Omega| \frac{1}{n^{2}} .
$$

Since

$$
\int_{\Omega_{n}^{+}}|\nabla u|^{r_{2}} u d x+\int_{\Omega_{n}^{-}}|\nabla u|^{r_{2}} u d x=\int_{\Omega}|\nabla u|^{r_{2}} u d x
$$


and

$$
\int_{\Omega}|\nabla u|^{r_{2}}|u| d x \leq\left(\int_{\Omega}|\nabla u|^{N} d x\right)^{r_{2} / N}\left(\int_{\Omega}|u|^{N /\left(N-r_{2}\right)} d x\right)^{\left(N-r_{2}\right) / N} \leq C\|u\|_{W_{0}^{1, N}(\Omega)}^{r_{2}+1} .
$$

Thus (3.5), (3.8) and (3.9) imply

$$
\begin{aligned}
\langle F(\xi), \xi\rangle & \geq\|u\|_{W_{0}^{1, N}(\Omega)}^{N}-\lambda\left(a_{1} C_{1}\|u\|_{W_{0}^{1, N}(\Omega)}^{r_{1}+1}+a_{2} C_{2}\|u\|_{W_{0}^{1, N}(\Omega)}^{r_{2}+1}\right) \\
& -C_{3}\|u\|_{W_{0}^{1, N}(\Omega)}^{r_{3}+1}\left(\int_{\Omega} \exp \left(N 2^{\frac{N}{N-1}} \alpha|u|^{\frac{N}{N-1}}\right) d x\right)^{\frac{1}{N}}-\frac{C_{4}}{n}\|u\|_{W_{0}^{1, N}(\Omega)} \\
& -\lambda a_{1}|\Omega| \frac{1}{n^{r_{1}+1}}-C_{5} \exp \left(2^{\frac{N}{N-1}} \alpha\right)|\Omega| \frac{1}{n^{2}}-|\Omega| \frac{1}{n^{2}} .
\end{aligned}
$$

Assume now that $\|u\|_{W_{0}^{1, N}(\Omega)}=r$ for some $r>0$ to be chosen later. We have

$$
\int_{\Omega} \exp \left(N 2^{\frac{N}{N-1}} \alpha|u|^{\frac{N}{N-1}}\right) d x=\int_{\Omega} \exp \left(N 2^{\frac{N}{N-1}} \alpha r^{\frac{N}{N-1}}\left(\frac{|u|}{\|u\|_{W_{0}^{1, N}(\Omega)}}\right)^{\frac{N}{N-1}}\right) d x
$$

and in order to apply the Trudinger-Moser inequality (1.4) we must have $N 2^{\frac{N}{N-1}} \alpha r^{\frac{N}{N-1}} \leq$ $\alpha_{N}$. Consequently,

$$
r \leq \frac{1}{2}\left(\frac{\alpha_{N}}{N \alpha}\right)^{\frac{N-1}{N}}
$$

Then

$$
\sup _{\|u\|_{W_{0}^{1, N}(\Omega)} \leq 1} \int_{\Omega} \exp \left(N 2^{\frac{N}{N-1}} \alpha r^{\frac{N}{N-1}}|u|^{\frac{N}{N-1}}\right) d x \leq L(N)|\Omega| .
$$

Hence,

$$
\begin{aligned}
\langle F(\xi), \xi\rangle & \geq r^{N}-\lambda\left(a_{1} C_{1} r^{r_{1}+1}+a_{2} C_{2} r^{r_{2}+1}\right)-C_{3} r^{r_{3}+1} L^{1 / N}(N)-\frac{C_{4}}{n} r \\
& -\lambda a_{1}|\Omega| \frac{1}{n^{r_{1}+1}}-C_{5} \exp \left(2^{\frac{N}{N-1}} \alpha\right)|\Omega| \frac{1}{n^{2}}-|\Omega| \frac{1}{n^{2}} .
\end{aligned}
$$

We need to choose $r$ such that

$$
r^{N}-C_{3} L(N)^{\frac{1}{N}} r^{r_{3}+1} \geq \frac{r^{N}}{2}
$$

in other words,

$$
r \leq \frac{1}{\left(2 C_{3} L(N)^{\frac{1}{N}}\right)^{\frac{1}{r_{3}+1-N}}}
$$

Thus, let $r=\min \left\{\frac{1}{2\left(2 C_{3} L(N)^{\frac{1}{N}}\right)^{\frac{1}{r_{3}+1-N}}}, \frac{1}{2}\left(\frac{\alpha_{N}}{N \alpha}\right)^{\frac{N-1}{N}}\right\}$, hence $\langle F(\xi), \xi\rangle \geq \frac{r^{N}}{2}-\lambda\left(a_{1} C_{1} r^{r_{1}+1}+a_{2} C_{2} r^{r_{2}+1}\right)-\frac{C_{4}}{n} r-\lambda a_{1}|\Omega| \frac{1}{n^{r_{1}+1}}-C_{5} \exp \left(2^{\frac{N}{N-1}} \alpha\right)|\Omega| \frac{1}{n^{2}}-|\Omega| \frac{1}{n^{2}}$.

Now, defining $\rho=\frac{r^{N}}{2}-\lambda\left(a_{1} C_{1} r^{r_{1}+1}+a_{2} C_{2} r^{r_{2}+1}\right)$, we choose $\lambda^{*}>0$ such that $\rho>0$ for $\lambda<\lambda^{*}$. Since $0<r_{i}<N-1$, for $i=1$, 2 , we can choose

$$
\lambda^{*}=\frac{1}{2} \frac{r^{N}}{2 a_{1} C_{1} r^{r_{1}+1}+2 a_{2} C_{2} r^{r_{2}+1}} .
$$


Now we choose $n^{*} \in \mathbb{N}$ such that

$$
\frac{C_{4}}{n} r+\lambda a_{1}|\Omega| \frac{1}{n^{r_{1}+1}}+C_{5} \exp \left(2^{\frac{N}{N-1}} \alpha\right)|\Omega| \frac{1}{n^{2}}+|\Omega| \frac{1}{n^{2}}<\frac{\rho}{2},
$$

for every $n \geq n^{*}$. Let $\xi \in \mathbb{R}^{m}$, such that $|\xi|_{m}:=\left\|\sum_{i=1}^{m} \xi_{i} w_{i}\right\|_{W_{0}^{1, N}(\Omega)}=r$, then for $\lambda<\lambda^{*}$ and $n \geq n^{*}$ we obtain

$$
\langle F(\xi), \xi\rangle \geq \frac{\rho}{2}>0 .
$$

Then by Lemma 3.1, for every $m \in \mathbb{N}$ there exists $y \in \mathbb{R}^{m}$ (with $|y|_{m} \leq r$ ) such that $F(y)=0$. Therefore, there exists $u_{m} \in W_{m}$ verifying

$$
\left\|u_{m}\right\|_{W_{0}^{1, N}(\Omega)} \leq r, \text { for every } m \in \mathbb{N}
$$

and such that

$$
\begin{aligned}
\int_{\Omega}\left|\nabla u_{m}\right|^{N-2} \nabla u_{m} \nabla w d x= & \lambda\left(a_{1} \int_{\Omega}\left(u_{m}^{+}\right)^{r_{1}} w d x+a_{2} \int_{\Omega}\left|\nabla u_{m}\right|^{r_{2}} w d x\right) \\
& +\int_{\Omega} f_{n}\left(\left(u_{m}\right)_{+}\right) w d x+\frac{1}{n} \int_{\Omega} w d x, \forall w \in W_{m} .
\end{aligned}
$$

Since $W_{m} \subset W_{0}^{1, N}(\Omega) \forall m \in \mathbb{N}$ and $r$ does not depend on $m$, then $\left(u_{m}\right)$ is a bounded sequence in $W_{0}^{1, N}(\Omega)$. Then, for some subsequence, there exists $u_{n} \in W_{0}^{1, N}(\Omega)$ (to simplify the notation, until the end of this section we will omit the subscript $n$ of the variable $u$ ) such that

$$
u_{m} \rightarrow u \text { weakly in } W_{0}^{1, N}(\Omega)
$$

and

$$
u_{m} \rightarrow u \text { in } L^{N}(\Omega) \text { and a.e. in } \Omega \text {. }
$$

Notice that

$$
\|u\|_{W_{0}^{1, N}(\Omega)} \leq \liminf _{m \rightarrow \infty}\left\|u_{m}\right\|_{W_{0}^{1, N}(\Omega)} \leq r, \forall n \in \mathbb{N},
$$

and $r$ does not depend on $n$. We claim that

$$
u_{m} \rightarrow u \text { in } W_{0}^{1, N}(\Omega) .
$$

Using the fact that $\mathcal{B}=\left\{w_{1}, w_{2}, \ldots, w_{n}, \ldots\right\}$ is a Schauder basis of $W_{0}^{1, N}(\Omega)$, for every $u \in W_{0}^{1, N}(\Omega)$ there exists a unique sequence $\left(\alpha_{n}\right)_{n \geq 1}$ in $\mathbb{R}$ such that $u=\sum_{j=1}^{\infty} \alpha_{j} w_{j}$, so

$$
\psi_{m}:=\sum_{j=1}^{m} \alpha_{j} w_{j} \rightarrow u \text { in } W_{0}^{1, N}(\Omega) \text { as } m \rightarrow \infty .
$$

Using as test function $w=\left(u_{m}-\psi_{m}\right) \in W_{m}$ in (3.14), we get

$$
\begin{aligned}
\int_{\Omega}\left|\nabla u_{m}\right|^{N-2} \nabla u_{m} \nabla\left(u_{m}-\psi_{m}\right) d x= & \lambda\left(a_{1} \int_{\Omega}\left(u_{m}^{+}\right)^{r_{1}}\left(u_{m}-\psi_{m}\right) d x+a_{2} \int_{\Omega}\left|\nabla u_{m}\right|^{r_{2}}\left(u_{m}-\psi_{m}\right) d x\right) \\
& +\int_{\Omega} f_{n}\left(\left(u_{m}\right)_{+}\right)\left(u_{m}-\psi_{m}\right) d x+\frac{1}{n} \int_{\Omega}\left(u_{m}-\psi_{m}\right) d x .
\end{aligned}
$$

By continuity of $f_{n},(3.15),(3.16),(3.19)$ and hypothesis $(F)$, we get 


$$
\begin{gathered}
\lim _{m \rightarrow \infty} \frac{1}{n} \int_{\Omega}\left(u_{m}-\psi_{m}\right) d x=0 \\
\lim _{m \rightarrow \infty} a_{1} \int_{\Omega}\left(u_{m}^{+}\right)^{r_{1}}\left(u_{m}-\psi_{m}\right) d x=0, \\
\lim _{m \rightarrow \infty} a_{2} \int_{\Omega}\left|\nabla u_{m}\right|^{r_{2}}\left(u_{m}-\psi_{m}\right) d x=0
\end{gathered}
$$

and

$$
\lim _{m \rightarrow \infty} \int_{\Omega} f_{n}\left(\left(u_{m}\right)_{+}\right)\left(u_{m}-\psi_{m}\right) d x=0 .
$$

Notice that (3.21), (3.22) and (3.23) are immediately. Let us verigy (3.24). By continuity of $f_{n}$ and (3.16) we obtain

$$
f_{n}\left(\left(u_{m}\right)_{+}\right)^{N^{\prime}} \rightarrow f_{n}\left(u_{+}\right)^{N^{\prime}} \text { a.e. in } \Omega
$$

and by Lemma 2.1 and (3.13), we obtain

$$
\int_{\Omega} f_{n}\left(\left(u_{m}\right)_{+}\right)^{N^{\prime}} d x \leq c_{n}^{N^{\prime}} \int_{\Omega}\left|u_{m}\right|^{N^{\prime}}=\left\|u_{m}\right\|_{L^{N^{\prime}}(\Omega)}^{N^{\prime}} \leq c_{n}^{N^{\prime}} C\left\|u_{m}\right\|_{W_{0}^{1, N}(\Omega)}^{N^{\prime}} \leq c_{n}^{N^{\prime}} C r^{N^{\prime}} .
$$

Hence, [16, Theorem 13.44] leads to

$$
f_{n}\left(\left(u_{m}\right)_{+}\right) \rightarrow f_{n}\left(u_{+}\right) \text {weakly in } L^{N^{\prime}}(\Omega) .
$$

Applying (3.16), (3.19) and (3.25), we conclude that (3.24) holds.

By (3.13) and (3.15), we obtain

$$
\lim _{m \rightarrow \infty} \int_{\Omega}\left|\nabla u_{m}\right|^{N-2} \nabla u_{m} \nabla\left(u-\psi_{m}\right) d x=0 .
$$

By $(3.21)-(3.24)$ and $(3.26)$, we obtain

$$
\lim _{m \rightarrow \infty} \int_{\Omega}\left|\nabla u_{m}\right|^{N-2} \nabla u_{m} \nabla\left(u_{m}-u\right) d x=0 .
$$

Now it is sufficient to apply the $\left(S_{+}\right)-$property of $-\Delta_{p}$ (see, e.g., [23, Proposition 3.5.]) for obtaining (3.18).

Let $k \in \mathbb{N}$, then for every $m \geq k$ we obtain

$$
\begin{aligned}
\int_{\Omega}\left|\nabla u_{m}\right|^{N-2} \nabla u_{m} \nabla w_{k} d x= & \lambda\left(a_{1} \int_{\Omega}\left(u_{m}^{+}\right)^{r_{1}} w_{k} d x+a_{2} \int_{\Omega}\left|\nabla u_{m}\right|^{r_{2}} w_{k} d x\right)+\int_{\Omega} f_{n}\left(\left(u_{m}\right)_{+}\right) w_{k} d x \\
& +\frac{1}{n} \int_{\Omega} w_{k} d x, \forall w_{k} \in W_{k} .
\end{aligned}
$$

Lettin $m \rightarrow \infty$, on accout of (3.18) we arrive at

$$
\begin{aligned}
\int_{\Omega}|\nabla u|^{N-2} \nabla u \nabla w_{k} d x= & \lambda\left(a_{1} \int_{\Omega}\left(u^{+}\right)^{r_{1}} w_{k} d x+a_{2} \int_{\Omega}|\nabla u|^{r_{2}} w_{k} d x\right)+\int_{\Omega} f_{n}\left(u_{+}\right) w_{k} d x \\
& +\frac{1}{n} \int_{\Omega} w_{k} d x, \quad \forall w_{k} \in W_{k} .
\end{aligned}
$$


Since $\left[W_{k}\right]_{k \in \mathbb{N}}$ is dense in $W_{0}^{1, N}(\Omega)$ we conclude that

$$
\begin{aligned}
\int_{\Omega}|\nabla u|^{N-2} \nabla u \nabla w d x= & \lambda\left(a_{1} \int_{\Omega}\left(u^{+}\right)^{r_{1}} w d x+a_{2} \int_{\Omega}|\nabla u|^{r_{2}} w d x\right)+\int_{\Omega} f_{n}\left(u_{+}\right) w d x \\
& +\frac{1}{n} \int_{\Omega} w d x, \quad \forall w \in W_{0}^{1, N}(\Omega) .
\end{aligned}
$$

Furthermore, $u \geq 0$ in $\Omega$. In fact, since $u_{-} \in W_{0}^{1, N}(\Omega)$ then from (3.2) we obtain

$$
\begin{aligned}
\int_{\Omega}|\nabla u|^{N-2} \nabla u \nabla u_{-} d x= & \lambda\left(a_{1} \int_{\Omega}\left(u^{+}\right)^{r_{1}} u_{-} d x+a_{2} \int_{\Omega}|\nabla u|^{r_{2}} u_{-} d x\right)+\int_{\Omega} f_{n}\left(u_{+}\right) u_{-} d x \\
& +\frac{1}{n} \int_{\Omega} u_{-} d x .
\end{aligned}
$$

Hence

$-\left\|u_{-}\right\|_{W_{0}^{1, N}(\Omega)}^{N}=\lambda\left(a_{1} \int_{\Omega}\left(u^{+}\right)^{r_{1}} u_{-} d x+a_{2} \int_{\Omega}|\nabla u|^{r_{2}} u_{-} d x\right)+\int_{\Omega} f_{n}\left(u_{+}\right) u_{-} d x+\frac{1}{n} \int_{\Omega} u_{-} d x \geq 0$,

because $\int_{\Omega} f_{n}\left(u_{+}\right) u_{-} d x=0$. Then $u_{-} \equiv 0$ a.e. in $\Omega$.

The first inequality in hypothesis $(F)$ and the equation in $\left(P_{n}\right)$ guarantee that $u \neq 0$. Here the presence of $\frac{1}{n}>0$ is needed. Next, we observe that hypothesis $(F)$ allows us to refer to $\left[18\right.$, Theorem 7.1] from which we infer that $u \in L^{\infty}(\Omega)$. Furthermore, the regularity result up to the boundary in [20, Theorem 1] and [21, p. 320] ensures that $u \in C^{1, \beta}(\bar{\Omega})$ with some $\beta \in(0,1)$. We also note that we may apply the strong maximum principle in $[24$, Theorem 5.4.1]. We are thus in a position to apply [24, Theorem 5.4.1] concluding that $u>0$ in $\Omega$ because we know that $u \geq 0, u \neq 0$, thereby $u$ is a solution of problem $\left(P_{n}\right)$. This completes the proof.

Remark 3.3. To apply [18, Theorem 7.1] and infer that $u \in L^{\infty}(\Omega)$, notice that it is necessary to consider the approximating functions $f_{n}$ (given by Lemma 2.1) instead of $f$. In fact, since

$$
F_{n}(v, p)=\lambda\left(a_{1} v^{r_{1}}+a_{2}|p|^{r_{2}}\right)+f_{n}(v)+\frac{1}{n}
$$

satisfies the inequality (7.2) in [18], because

$$
\operatorname{sign}(v) \cdot F_{n}(v, p) \leq \lambda\left(a_{1}|v|^{r_{1}}+a_{2}|p|^{r_{2}}\right)+c_{n}|v|+\frac{1}{n} .
$$

While $F(v, p)=\lambda\left(a_{1} v^{r_{1}}+a_{2}|p|^{r_{2}}\right)+f(v)$ does not necessarily satisfy such a hypothesis, in fact

$$
\operatorname{sign}(v) . F(v, p) \leq \lambda\left(a_{1}|v|^{r_{1}}+a_{2}|p|^{r_{2}}\right)+a_{3}|u|^{r_{3}} \exp \left(\alpha|v|^{\frac{N}{N-1}}\right) .
$$

\section{Proof of the MAin Result}

In this section we will prove Theorem 1.1. Consider the following problem

$$
\left\{\begin{array}{lll}
-\Delta_{N} v=\lambda a_{1} v^{r_{1}} & \text { in } & \Omega \\
v>0 & \text { in } & \Omega \\
v=0 & \text { on } & \partial \Omega
\end{array}\right.
$$

where $\lambda, a_{1}$ and $r_{1}$ were given in Theorem 1.1. This problem admits a solution $v_{0} \in C_{0}^{1}(\bar{\Omega})$, see for instace [13, Lemma 4.1]. The function $v_{0}$ allows us to bound from below the solutions of $\left(P_{n}\right)$. 
For each $\lambda \in\left(0, \lambda^{*}\right)$ and $n \in \mathbb{N}$ sufficiently large, by using Lemma 3.2, we get that equation $\left(P_{n}\right)$ has a weak solution $u_{n} \in W_{0}^{1, N}(\Omega) \cap C^{1, \alpha}(\bar{\Omega})$ for some $\alpha \in(0,1)$.

In view of (3.17), we can argue as for (3.18), to find a subsequence $n \rightarrow \infty$ such that the corresponding sequence $\left\{u_{n}\right\}$ is strongly convergent:

$$
u_{n} \rightarrow u \text { in } W_{0}^{1, N}(\Omega) .
$$

In fact, for some subsequence, there exists $u \in W_{0}^{1, N}(\Omega)$ such that

$$
u_{n} \rightarrow u \text { weakly in } W_{0}^{1, N}(\Omega)
$$

and, by Sobolev embedding for $1 \leq s<+\infty$,

$$
u_{n} \rightarrow u \text { in } L^{s}(\Omega) \text { and a.e. in } \Omega \text {. }
$$

Notice that

$$
\int_{\Omega}\left|\nabla u_{n}\right|^{N-2} \nabla u_{n} \nabla w \geq \lambda a_{1} \int_{\Omega}\left(u_{n}\right)^{r_{1}} w, \forall w \in W_{0}^{1, N}(\Omega) \text { with } w \geq 0 .
$$

Since

$$
u_{n} \rightarrow u \text { a.e. in } \Omega \text {, }
$$

we have

$$
f_{n}\left(u_{n}(x)\right) \rightarrow f(u(x)) \text { a.e. in } \Omega,
$$

by the uniform convergence of Lemma 2.1 (iii).

By Lemma 2.2

$$
\begin{aligned}
\int_{\Omega} & f_{n}\left(u_{n}\right)^{N^{\prime}} d x=\int_{\Omega_{n}^{+}} f_{n}\left(u_{n}\right)^{N^{\prime}} d x+\int_{\Omega_{n}^{-}} f_{n}\left(u_{n}\right)^{N^{\prime}} d x \\
\int_{\Omega_{n}^{+}} f_{n}\left(u_{n}\right)^{N^{\prime}} d x & \leq C_{1}^{\frac{N}{N-1}} \int_{\Omega_{n}^{+}}\left|u_{n}\right|^{\left(r_{3}-1\right) \frac{N}{N-1}} \exp \left(\frac{N}{N-1} 2^{\frac{N}{N-1}} \alpha\left|u_{n}\right|^{\frac{N}{N-1}}\right) d x \\
& \leq C_{1}^{\frac{N}{N-1}}\left(\int_{\Omega}\left|u_{n}\right|^{\left(r_{3}-1\right) \frac{N}{N-2}} d x\right)^{\frac{N-2}{N-1}}\left(\int_{\Omega} \exp \left(N 2^{\frac{N}{N-1}} \alpha\left|u_{n}\right|^{\frac{N}{N-1}}\right) d x\right)^{\frac{1}{N-1}} \\
& =C_{1}^{\frac{N}{N-1}}\left\|u_{n}\right\|_{L^{\left(r_{3}-1\right)}}^{\left(r_{3}-1\right) \frac{N}{N-2}}(\Omega) \\
& \left.\left.\leq C\left\|u_{n}\right\|_{W_{0}^{1, N}(\Omega)}^{\left(r_{3}-1\right) \frac{N}{N-1}}\left(\int_{\Omega} \exp \left(N 2^{\frac{N}{N-1}} \alpha\left|u_{n}\right|^{\frac{N}{N-1}}\right) d x\right)^{\frac{N}{N-1}} \alpha\left|u_{n}\right|^{\frac{N}{N-1}}\right) d x\right)^{\frac{1}{N-1}}
\end{aligned}
$$

and

$$
\begin{aligned}
\int_{\Omega_{n}^{-}} f_{n}\left(u_{n}\right)^{N^{\prime}} d x & \leq C_{2}^{\frac{N}{N-1}} \int_{\Omega_{n}^{-}}\left|u_{n}\right|^{\frac{N}{N-1}} \exp \left(\frac{N}{N-1} 2^{\frac{N}{N-1}} \alpha\left|u_{n}\right|^{\frac{N}{N-1}}\right) d x \\
\leq & C_{2}^{\frac{N}{N-1}} \exp \left(\frac{N}{N-1} 2^{\frac{N}{N-1}} \alpha\right)|\Omega| \frac{1}{n^{\frac{N}{N-1}}} .
\end{aligned}
$$

Since $\left\|u_{n}\right\|_{W_{0}^{1, N}(\Omega)} \leq r$, by the estimates before, we obtain

$$
\int_{\Omega} f_{n}\left(u_{n}\right)^{N^{\prime}} d x \leq C
$$

for each $n$. Since $f_{n}\left(u_{n}(x)\right) \rightarrow f(u(x))$ a.e. in $\Omega$, [16, Theorem 13.44] leads to

$$
f_{n}\left(u_{n}\right) \rightarrow f(u) \text { weakly in } L^{N^{\prime}}(\Omega) .
$$


Recall from (3.2) that, forall $w \in W_{0}^{1, N}(\Omega)$,

$$
\int_{\Omega}\left|\nabla u_{n}\right|^{N-2} \nabla u_{n} \nabla w=\lambda\left(a_{1} \int_{\Omega}\left(u_{n}\right)^{r_{1}} w+a_{2} \int_{\Omega}\left|\nabla u_{n}\right|^{r_{2}} w\right)+\int_{\Omega} f_{n}\left(u_{n}\right) w+\frac{1}{n} \int_{\Omega} w .
$$

Taking $w=u_{n}-u$ in (4.7), we obtain

$$
\lim _{m \rightarrow \infty} \int_{\Omega}\left|\nabla u_{n}\right|^{N-2} \nabla u_{n} \nabla\left(u_{n}-u\right) d x=0 .
$$

Now it is sufficient to apply the $\left(S_{+}\right)$- property of $-\Delta_{p}$ for obtaining (4.2).

Then from (4.2) and (4.6) and the fact that $u_{n}$ solves $\left(P_{n}\right)$, by passing to limit when $n \rightarrow+\infty$ we get that

$$
\int_{\Omega}|\nabla u|^{N-2} \nabla u \nabla w=\lambda\left(a_{1} \int_{\Omega}(u)^{r_{1}} w+a_{2} \int_{\Omega}|\nabla u|^{r_{2}} w\right)+\int_{\Omega} f(u) w, \quad \forall w \in W_{0}^{1, N}(\Omega) .
$$

Now, we are going to check that $u>0$ in $\Omega$. Notice that, by (4.1) and (4.4), for each $n$ sufficiently large $u_{n}$ is a supersolution and $v_{0}$ is a subsolution of Problem (4.1). In order to apply Theorem 2.3, we need to check that $\frac{u_{n}}{v_{0}}, \frac{v_{0}}{u_{n}} \in L^{\infty}(\Omega)$. This follows by using Hopf boundary point lemma (in the strong maximum principle for both Dirichlet problems $(4.1)$ and $\left(P_{n}\right)$ with corresponding solutions $v_{0}$ and $\left.u_{n}\right)$, regularity up to the boundary and L'Hôpital theorem (see [13] for details). Therefore, $u_{n}(x) \geq v_{0}(x)>0$ for all $x \in \Omega$. Thus, by passing to the limit, we conclude that $u$ is a positive solution of problem $(P)$ and the proof of the theorem is thus complete.

\section{REFERENCES}

[1] C. O. Alves and D. G. de Figueiredo, Nonvariational Elliptic Systems via Galerkin Methods, D. Haroske, T. Runst and H. J. Schmeisser (eds.) Function Spaces, Differential Operators and Nonlinear Analysis. The Hans Triebel Anniversary Volume, 2003

[2] H. Amann, M.G. Crandall, On some existence theorems for semi-linear elliptic equations, Indiana Univ. Math. J. 27 (1978) 779-790.

[3] F. V. Atkinson and L. A. Peletier, Elliptic equations with critical growth. Math. Inst. Univ. Leiden, Rep. $21(1986)$

[4] H. Brézis, Functional analysis, Sobolev spaces and partial differential equations, (Springer, 2011).

[5] H. Brézis, R.E.L. Turner, On a class of superlinear elliptic problems, Comm. Partial Differential Equations 2 (1977) 601614.

[6] A.L.A. de Araujo, M. Montenegro, Existence of solution for a general class of elliptic equations with exponential growth, Ann. Mat. 195 (2016) 1737-1748.

[7] A.L.A. de Araujo, M. Montenegro, Existence of solution for a nonvariational elliptic system with exponential growth in dimension two. J. Differential Equations, v. 264 (2018) 2270-2286.

[8] D.G. de Figueiredo, M. Girardi and M. Matzeu, Semilinear elliptic equations with dependence on the gradient via mountain-pass techniques, Dier. Integr. Equ., 17 (2004), 119-126.

[9] de Figueiredo, D.G., do Ó, J.M., Ruf, B.: On an inequality by N. Trudinger and J. Moser and related elliptic equations. Commun. Pure Appl. Math. 55, 135152 (2002)

[10] de Figueiredo, D.G., Miyagaki, O.H., Ruf, B.: Elliptic equations in R2 with nonlinearities in the critical growth range. Calc. Var. Partial Differ. Equ. 3, 139153 (1995) 6

[11] L.R. de Freitas, Multiplicity of solutions for a class of quasilinear equations with exponential critical growth. Nonlinear Anal. TMA 95, 607624 (2014) 1

[12] F. Faraci, D. Motreanu and D. Puglisi, Positive solutions of quasi-linear elliptic equations with dependence on the gradient, Calc. Var. Partial Dierential Equations, 54 (2015), 525-538.

[13] L.F.O Faria, O.H. Miyagaki, D. Motreanu, Comparison and positive solutions for problemswith (p, q)-Laplacian and convection term. Proc. Edinb. Math. Soc. 57(2), 687-698 (2014). 
[14] L. F. O. Faria, O. H. Miyagaki, D. Motreanu and M. Tanaka, Existence results for nonlinear elliptic equations with Leray-Lions operator and dependence on the gradient, Nonlinear Anal., 96 (2014), 154166.

[15] S. Fučík, O. John, J. Nečas, On the existence of Schauder bases in Sobolev spaces. Comment. Math. Univ. Carol. 13, 163-175 (1972)

[16] E. Hewitt and K. Stromberg, Real and Abstract Analysis, SpringerVerlag, 1975.

[17] S. Kesavan, Topics in functional analysis and applications, John Wiley and Sons, (1989).

[18] O. A. Ladyzhenskaya and N. N. Ural'tseva, Linear and quasilinear elliptic equations (Academic Press, 1968).

[19] N.Lam, G. Lu, Elliptic equations and systems with subcritical and critical exponential growth without the AmbrosettiRabinowitz condition. J. Geom. Anal. 24, 118143 (2014)

[20] G. M. Lieberman, Boundary regularity for solutions of degenerate elliptic equations, Nonlinear Anal. 12 (1988) 1203-1219.

[21] G. M. Lieberman, The natural generalization of the natural conditions of Ladyzhenskaya and Ural'tseva for elliptic equations, Comm. Partial Differential Equations 16 (1991), 311-361.

[22] J. Moser, A sharp form of an inequality by Trudinger. Indiana Univ. Math. J. 20 1077-1092 (1971)

[23] D. Motreanu, V.V. Motreanu AND N.S. Papageorgiou, Multiple constant sign and nodal solutions for Nonlinear Neumann eigenvalue problems, Ann. Sc. Norm. Sup. Pisa Cl. Sci. (5), X (2011), 1-27.

[24] P. Pucci AND J. Serrin, The maximum principle, (Birkhuser Verlag, 2007).

[25] D. Ruiz, A priori estimates and existence of positive solutions for strongly nonlinear problems, J. Differential Equations 199 (2004) 96114.

[26] E.A.B. Silva, S.H.M. Soares, Liouville-Gelfand type problems for the N-Laplacian on bounded domains of $R^{N}$. Annali della Scuola Normale Superiore di Pisa. Classe di Scienze 4, 130 (1999)

[27] W. A. Strauss, On weak solutions of semilinear hyperbolic equations, An. Acad. Brasil. Ciênc. 42 645-651 (1970)

[28] N. S. Trudinger, On the imbeddings into Orlicz spaces and applications. J. Math. Mech. 17 473-484 (1967)

[29] Y. Yang and K. Perera, (N, q)-Laplacian problems with critical TrudingerMoser nonlinearities, Bull. London Math. Soc. 48 (2016), 260270.

2

Universidade Federal de Viçosa, Departamento de Matemática, Avenida Peter Henry Rolfs, S/N, CEP 36570-900, ViÇOSA, MG, BRAZIL

E-mail address: anderson.araujo@ufv.br

Departamento de Matemática, Universidade Federal de Juiz de Fora, CEP 30161-970, Juiz DE FORA - MG, BRAZIL

E-mail address: luiz.faria@ufjf.edu.br 